\title{
EXPRESIÓN DE METALOPROTEASA DE MATRIZ 9 EN EL CÁNCER DE PRÓSTATA. EXPERIENCIA PRELIMINAR
}

Iván González Rodríguez, Manuel Rivas del Fresno, Rodrigo Gil Ugarteburu, Patricia González Arriaga, Felicitas López Cima, Ana Fernández Samoano, Valentín Muruamendiaraz Fernández, Isabel Fernández García, Jose Ramón Pérez-Carral y Adonina Tardón.

Servicio de Urología. Hospital de Cabueñes. Gijón. Asturias. España.

Resumen.- OBJETIVO: Estudiar la validez de la metaloproteasa 9 (MMP-9) como marcador complementario al PSA en el diagnóstico y el pronóstico del carcinoma de próstata.

MÉTODO: Estudio prospectivo estructurado como cohorte de base hospitalaria. Fueron incluidos 100 pacientes consecutivos a los que se iba a practicar una biopsia prostática. La determinación sérica de MMP-9 se realizó mediante inmunoensayo, y el análisis estadístico con el programa informático stata/SE 8.2.

RESULTADOS: 32 pacientes fueron diagnosticados de carcinoma prostático y el 52\% de ellos con grado Gleason mayor o igual a 7. Los valores de MMP-9 sérica os-

\section{CORRESPONDENCIA}

Iván González Rodríguez

Despacho de Urología

Hospital de Cabueñes

Avenida de los Prados, 395

Gijón. Asturias. (España).

elivanin@yahoo.es

Aceptado para publicar: 24 de marzo 2009. cilaron entre 225,7 y 1932,3 nanogramos por mililitro, sin encontrar diferencias estadísticamente significativas entre los pacientes con histología benigna, maligna e incierta $(p=0,429)$. Las diferencias se acercaron a la significación estadística en el subgrupo de pacientes con PSA 4-10 ng/ml (p=0,058) y en el subgrupo PSA libre/total menor de $15 \%$ se observaron diferencias significativas $(p=0,037)$. No se encontró relación entre el grado Gleason y el nivel de MMP-9 ( $p=0,739)$. Los niveles de PSA y MMP-9 demostraron ser independientes (Coeficiente de correlación de Pearson -0, 1).

CONCLUSIONES: No fue posible demostrar la eficacia de la MMP-9 para predecir el resultado de la biopsia. En el grupo de pacientes con elevaciones discretas del PSA (entre 4 y $10 \mathrm{ng} / \mathrm{ml}$ ) todas las variables descriptivas fueron superiores en el grupo con histología maligna, sin alcanzar la significación estadística. Sí se alcanzó la significación cuando el cociente de PSA libre entre PSA total fue menor del 15\%, pero este hallazgo no tiene relevancia en la práctica clínica, pues estos pacientes ya tienen indicación clara de biopsia. Tampoco se demuestra relación con el pronóstico al no existir diferencias de expresión de MMP-9 entre diferentes grados Gleason.

Palabras clave: Cáncer de Próstata. PSA. Diagnóstico. Marcadores. Metaloproteasa-9.

Summary.- OBJECTIVES: To study the validity of Matrix Metalloproteinase 9 as a complementary marker to PSA for the diagnosis and prognosis of Prostate Cancer.

METHODS: Prospective study structured as a hospitalbased cohort of 100 consecutive patients undergoing prostate biopsy. Serum determination of MMP-9 was 
carried out by means of inmunoassay. Statistical analysis was performed using the Stata/SE 8.2 software.

RESULTS: 32 patients were diagnosed with prostate cancer and 52\% had a Gleason score equal to or greater than 7. The values of serum MMP-9 varied between 225.7 and $1932.3 \mathrm{ng} / \mathrm{ml}$, without significant differences among patients with benign, malignant and uncertain histology $(p=0.429)$. The differences approached statistical significance in the subgroup of patients with PSA at $4-10 \mathrm{ng} / \mathrm{ml}(\mathrm{p}=0.058)$, and significant differences were observed in the subgroup with free PSA to total PSA coefficient of less than 15\% $(p=0.037)$. No relationship between the Gleason score and the level of MMP-9 was shown ( $p=0.739$ ). The levels of PSA and MMP-9 were shown to be independent (Pearson coefficient of correlation -0.1).

CONCLUSIONS: It was not possible to show the efficacy of MMP-9 in predicting the result of the biopsy. In the group of patients with slightly increased levels of PSA (between 4 and $10 \mathrm{ng} / \mathrm{mll}$ all the descriptive variables were higher in the group with malignant histology, though they did not reach statistical significance, they did reach significance when the coefficient of free PSA over total PSA was less than 15\%, but this finding is not relevant clinically, as these patients already have a clear indication for biopsy. Neither was the relationship with the prognosis shown as there are no differences of MMP-9 expression at varying Gleason scores.

Keywords: Prostate cancer, PSA, Diagnostic, Markers, Metalloproteinase-9.

\section{INTRODUCCIÓN}

Actualmente el diagnóstico de sospecha del cáncer de próstata se basa en la determinación sérica del Antígeno Prostático Específico (PSA), que en realidad resulta un marcador de valor limitado para esta función, con tan sólo un $30 \%$ de valor predictivo positivo $(1,2)$. Esto obliga a la realización de un gran número de biopsias prostáticas innecesarias. Por otra parte la heterogénea historia natural de la enfermedad plantea la duda del sobretratamiento tanto en pacientes no curables como en aquellos con tumores clínicamente no significativos.

En la carrera científica abierta para desarrollar nuevos marcadores diagnósticos y pronósticos del cáncer de próstata, las metaloproteasas llevan siendo estudiadas desde hace varios años, con resultados diversos. Las metaloproteasas de matriz son una familia de proteasas de gran complejidad funcional
(3). Intervienen en procesos de remodelación tisular como la embriogénesis, el desarrollo y la reparación de tejidos dañados (4), y su desregulación es causa de múltiples patologías como la artritis reumatoide, enfermedades autoinmunes y cardiopatías (5). Parecen jugar también un importante papel en la muerte celular programada y la formación de neovasos (6), fundamentales en el proceso tumoral, y gran número de estudios clínicos relacionan su actividad y expresión tisular o sérica con neoplasias de mama $(7,8)$, pulmón (9), colon (10), vejiga (11) o riñón (12).

La metaloproteasa de matriz 9 (MMP-9) degrada el colágeno desnaturalizado de las membranas basales y el intersticio celular, ejerciendo un papel clave en los procesos de crecimiento y diseminación tumoral. Este aspecto es especialmente interesante en el cáncer de próstata, pues podría segregar los tumores indolentes de los agresivos clínicamente.

En estudios previos ha sido demostrada una mayor actividad tisular de la MMP-9 en tejido prostático maligno frente a tejido hiperplásico (13). Ha sido demostrado también un aumento de expresión sérico del mRNA de la MMP-9 en pacientes con carcinoma prostático frente a pacientes sin tumor (14). Por otra parte, se ha observado un incremento sérico de MMP-9 en pacientes con cáncer de próstata metastático frente a pacientes con carcinoma localizado (15).

La hipótesis de este estudio es que existen diferencias en los niveles sanguíneos de MMP-9 entre los pacientes con cáncer prostático y los pacientes con histología benigna. También deberían existir diferencias entre pacientes con tumores prostáticos agresivos y silentes; y por tanto la determinación de los niveles sanguíneos de MMP-9 podría complementar al PSA como instrumento diagnóstico y pronóstico.

\section{MATERIAL Y MÉTODOS}

El estudio fue diseñado como una cohorte de base hospitalaria en la que fueron incluidos 100 pacientes consecutivos sospechosos de padecer un carcinoma prostático, y a los que iba a realizarse una biopsia prostática, entre octubre del 2005 y febrero de 2006, al cumplir al menos uno de los siguientes criterios de inclusión:

- PSA sérico superior a $4 \mathrm{ng} / \mathrm{ml}$

- Tacto rectal sospechoso de tumor prostático

- Imagen sospechosa de tumor en ecografía transrectal 
TABLA I. VALORES SÉRICOS DE METALOPROTEASA 9 MEDIDOS EN NANOGRAMOS POR MILIIITRO, SEGÚN LA PRESENCIA O AUSENCIA DE CARCINOMA PROSTÁTICO.

\begin{tabular}{|c|c|c|c|c|c|c|c|c|c|c|}
\hline $\begin{array}{c}\text { Tumor } \\
\text { maligno }\end{array}$ & $\mathbf{N}$ & Media & Desv Típ. & Mín. & P 25\% & P 50\% & P 75\% & Máx. & \multicolumn{2}{|c|}{$\begin{array}{c}\text { Kruskall -Wallis } \\
\text { p-valor }\end{array}$} \\
\hline No & 58 & 800.47 & 387.22 & 225.7 & 516.2 & 680.85 & 1055.4 & 1932.3 & \multirow{2}{*}{0.846} & \multirow{3}{*}{0.429} \\
\hline Si & 32 & 837.07 & 425.29 & 319.9 & 473.55 & 736.25 & 1240.1 & 1888 & & \\
\hline Otro & 5 & 998.44 & 402.35 & 632.3 & 789.5 & 877.1 & 1020.6 & 1672.7 & & \\
\hline
\end{tabular}

Para evitar sesgos, fueron excluidos los pacientes que cumplían al menos uno de los siguientes criterios:

- Denegación del consentimiento.

- Diagnóstico previo de neoplasia.

- Padecer enfermedades inflamatorias que alteren los niveles de metaloproteasas.

- Tratamiento en los 6 meses previos con fármacos que alteren el PSA.

La biopsia transrectal fue realizada por varios urólogos con experiencia en el procedimiento, tomando al menos 6 cilindros con una aguja "tru-cut" 18 Gauge, guiada por una sonda ecográfica de 7 $\mathrm{MHz}$ y un ecógrafo Siemens SONOLINE SI 250.

La extracción de sangre se realizó justo antes de la biopsia en tubos de plástico de $8 \mathrm{ml} Z$ Serum Sep Clot Activator Vacuette. Tras un periodo en nevera de entre 1 y 6 horas, las muestras fueron centrifugadas a 3000 r.p.m. durante 15 minutos, para congelarse inmediatamente a $-80^{\circ} \mathrm{C}$. La determinación del nivel sérico de MMP-9 fue realizada por personal especializado en el laboratorio de la unidad de Epidemiología Molecular del Instituto Asturiano de Oncología de la Universidad de Oviedo, mediante la técnica de ELISA (sándwich enzime inmunoassays) utilizando un set comercial Quantikine (DMP 900, R\&D Systems, Minneapolis USA) para realizar un inmunoensayo tipo sándwich. La determinación del PSA se realizó en el laboratorio de análisis clínicos del Hospital de Cabueñes según la técnica estándar de quimioluminiscencia (Advia Centaur PSA Ready Pack, Bayer).

El análisis estadístico se realizó con el soporte del programa informático Stata/SE 8.2. Se utilizaron estadísticos descriptivos para las variables continuas. La comparación entre variables categóricas se realizó mediante tablas de contingencia con el test $\chi 2$ de Pearson, así como el coeficiente de correlación de Pearson para el estudio de correlación. Se con- sideró la existencia de diferencias estadísticamente significativas cuando el p-valor fue menor de 0,05.

\section{RESULTADOS}

Entre los 100 pacientes biopsiados, con edades comprendidas entre 46 y 81 años, se diagnosticaron 34 cánceres de próstata y 6 lesiones dudosas (PIN o ASAP). El grado Gleason de los tumores fue determinado en 31 pacientes, con 6,5\% grados Gleason 4-5, 42\% grado Gleason 6, 29\% grado Gleason 7 y 22,5\% grados Gleason 8-9. Se excluyó a 3 pacientes del análisis, 2 por presencia de PSA muy alejados de la media y mediana de la serie (165 y $299 \mathrm{ng} / \mathrm{ml}$ ) y el otro por fractura de los tubos de sangre durante el procesamiento.

Los pacientes con resultado benigno en la biopsia presentaron un PSA medio de $9,26 \mathrm{ng} / \mathrm{ml}$ $(2,5-28,3)$, y los que fueron diagnosticados de carcinoma prostático $19,11 \mathrm{ng} / \mathrm{ml}(4,7-82,8)$. Las diferencias de PSA fueron estadísticamente significativas en pacientes con y sin carcinoma prostático $(p=0,030)$.

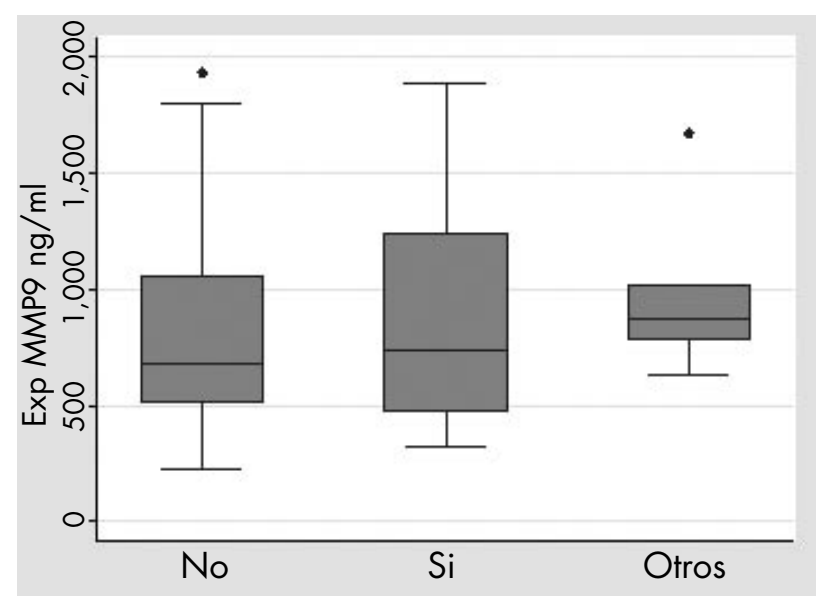

FIGURA 1. Expresión sérica de metaloproteasa 9, según la presencia o ausencia de carcinoma prostático. 
TABLA II. VALORES SÉRICOS DE METALOPROTEASA 9 (en $\mathrm{ng} / \mathrm{m} /$ ) DEL SUBGRUPO DE PACIENTES CON PSA 4-10 $\mathrm{ng} / \mathrm{ml}$.

\begin{tabular}{|c|c|c|c|c|c|c|c|c|c|}
\hline $\begin{array}{l}\text { Tumor } \\
\text { maligno }\end{array}$ & $\mathbf{N}$ & Media & Desv Típ. & Mín. & P 25\% & P $50 \%$ & P 75\% & Máx. & $\begin{array}{r}\text { Krusk } \\
p\end{array}$ \\
\hline No & 41 & 771.84 & 360.38 & 225.7 & 516.2 & 674.5 & 1011.1 & 1799.4 & \\
\hline $\mathrm{Si}$ & 15 & 987.75 & 428.56 & 366.3 & 704 & 953.1 & 1350.9 & 1888 & \\
\hline Otro & 5 & 998.44 & 402.35 & 632.3 & 789.5 & 877.1 & 1020.6 & 1672.7 & \\
\hline
\end{tabular}

Para el grupo de pacientes con PSA 4-10 $\mathrm{ng} / \mathrm{ml}$, el cociente PSA libre/total fue significativamente menor $(p=0,028)$ en los pacientes con cáncer de próstata (media $8,66 \%$ ), que en los pacientes sin cáncer de próstata media $(11,8 \%)$. Pero al utilizar el punto de corte del 15\%, los resultados no son significativos $(p=0,838)$.

En cuanto a la MMP-9, se obtuvieron datos de 95 pacientes. Para aquellos con tumor maligno, la media fue de $837,7 \mathrm{ng} / \mathrm{ml}(425,29-1888)$, para los pacientes con histología benigna la media fue de $800,47 \mathrm{ng} / \mathrm{ml}(225,7-1932,3)$. Para los pacientes con lesiones dudosas o premalignas la media fue de $998,44 \mathrm{ng} / \mathrm{ml}(402,35-1672,7)$. No se encontraron diferencias estadísticamente significativas en la expresión de metaloproteasas, $p=0,429$.

Agrupando a los pacientes diagnosticados de cáncer prostático según el grado Gleason de la biopsia, no fueron encontradas diferencias significativas en la expresión de MMP-9 entre los grupos de Gleason bajo, medio y alto $(p=0,739)$.

En el subgrupo de pacientes con PSA 4-10 $\mathrm{ng} / \mathrm{ml}$ los pacientes con cáncer de próstata no demostraron estadísticamente mayor expresión de MMP-9 que los pacientes sin tumor maligno $(p=0,058)$, si bien los valores estadísticos descriptivos fueron sistemáticamente menores en el grupo sin tumor maligno. En aquellos con PSA 4-10 ng/ml y cociente PSA libre/total menor del 15\%, los pacientes diagnosticados de cáncer prostático presentaron valores significativamente mayores de MMP-9 sérica $(p=0,037)$.

Se realizó un estudio de correlación entre la expresión de PSA y la de MMP-9 en los pacientes diagnosticados de cáncer de próstata, con el resultado de un Coeficiente de correlación de Pearson de $-0,1000$, por lo que fue asumido que los niveles de MMP-9 no guardan relación lineal con los de PSA séricos.

\section{DISCUSIÓN}

La generalización en el uso del PSA como marcador diagnóstico del cáncer de próstata ha supuesto un incremento progresivo de la incidencia del tumor en pacientes más jóvenes y en estadios más precoces. Pero esto se ha conseguido a costa de incrementar las biopsias prostáticas de manera exponencial, siendo éste un procedimiento no exento de riesgos y morbi-mortalidad (16). Según nuestros resultados, para el diagnóstico de un cáncer prostático son necesarias en nuestro estudio alrededor de 3 biopsias, dato acorde con la literatura existente desde el uso del PSA como marcador del diagnóstico de sospecha (17).

Entre los tumores detectados hay una clara prevalencia de los tumores de alto potencial maligno, expresados como grados Gleason medios y sobre todo altos. Tan solo un paciente fue diagnosticado

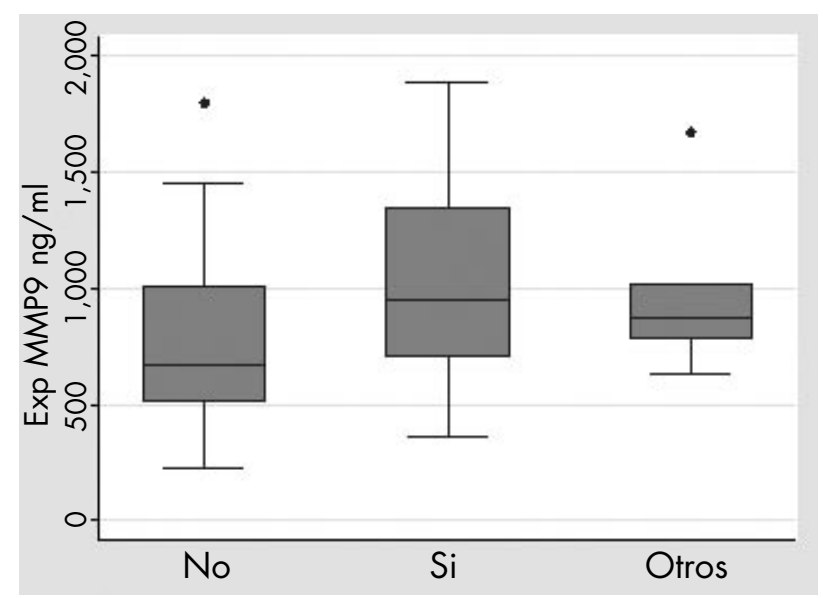

FIGURA 2. Expresión sérica de metaloproteasa 9 en el subgrupo de pacientes con PSA 4-10 ng/ml. 
TABLA III. VALORES SÉRICOS DE METALOPROTEASA 9 EN EL SUBGRUPO DE PACIENTES CON COCIENTE DE PSA LIBRE ENTRE PSA TOTAL MENOR DE 15\%.

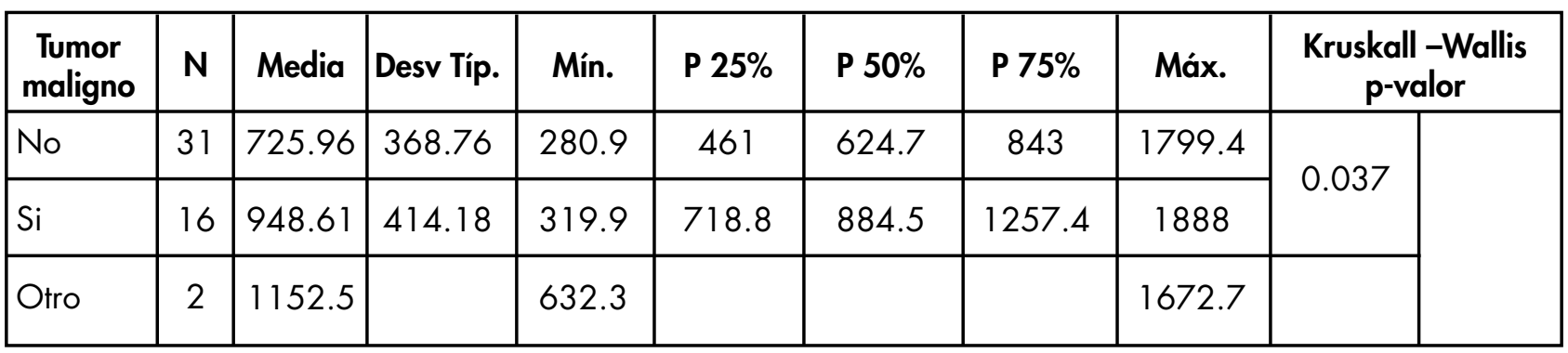

con un grado Gleason bajo. Todo ello concuerda con otros estudios, y nos permite extraer dos conclusiones. En primer lugar, la muestra parece representativa de la población estudiada, pero, por otra parte, debido a su escaso tamaño, dificulta la obtención de resultados estadísticos. Los pacientes diagnosticados de neoplasia prostática intraepitelial (PIN) y proliferación acinar atípica (ASAP) fueron incluidos juntos en un grupo aparte, pues aunque anatomopatológicamente no guardan relación, avalan la presencia sincrónica de un carcinoma prostático y en la práctica clínica suelen ser manejados de igual modo (18).

En cuanto al valor del PSA como marcador, en nuestro estudio no fueron encontrados tumores en pacientes con PSA menor de 4. Por otra parte también fue demostrado que al aumentar el PSA el riesgo de padecer un cáncer prostático fue mayor. En el subgrupo de pacientes con PSA de dudoso rango patológico (4-10), suelen utilizarse otros datos que aumenten el valor predictivo positivo del PSA y eviten biopsias innecesarias. Una de las medidas más utilizadas para decidir la biopsia es el cociente del PSA libre entre el PSA total. En el presente estudio se demuestra su valor estadístico como variable continua, pero sin significación estadística al utilizar el punto de corte del $15 \%$. Este resultado es dudosamente extrapolable a la población general por el escaso tamaño muestral y el sesgo de selección de los pacientes.

En este estudio no fue demostrada relación estadística entre el nivel de MMP-9 y el resultado histológico de la biopsia (benigno, incierto, maligno) posiblemente debido también al escaso tamaño muestral, pues los valores estadísticos descriptivos de la expresión de MMP-9 como el mínimo, la mediana y la moda son superiores en el grupo con cáncer de próstata.

En el estudio de los subgrupos según niveles de PSA se observa un acercamiento a la significación estadística en el rango clínicamente más proble- mático, aquel con PSA poco elevado (con variables descriptivas sistemáticamente superiores en los pacientes con tumor), e incluso llega a la significación estadística en el subgrupo de pacientes con cociente de PSA libre/total menor del 15\%, aunque este resultado aislado no tiene importancia clínica, pues estos pacientes iban a ser biopsiados en todo caso.

En el estudio de subgrupos según el Gleason de la biopsia, no se encontraron diferencias significativas en los niveles de metaloproteasas. Cabe destacar sin embargo la heterogeneicidad de los hallazgos ligada a grados Gleason altos, y el pequeño tamaño muestral (tan solo un Gleason bajo), que dificultan la presencia de resultados. No fue posible por tanto demostrar asociación entre la agresividad tumoral (evaluada según el patrón histopatológico) con el nivel de MMP-9. Debido a la estrecha relación entre el pronóstico de la enfermedad y el grado Glea-

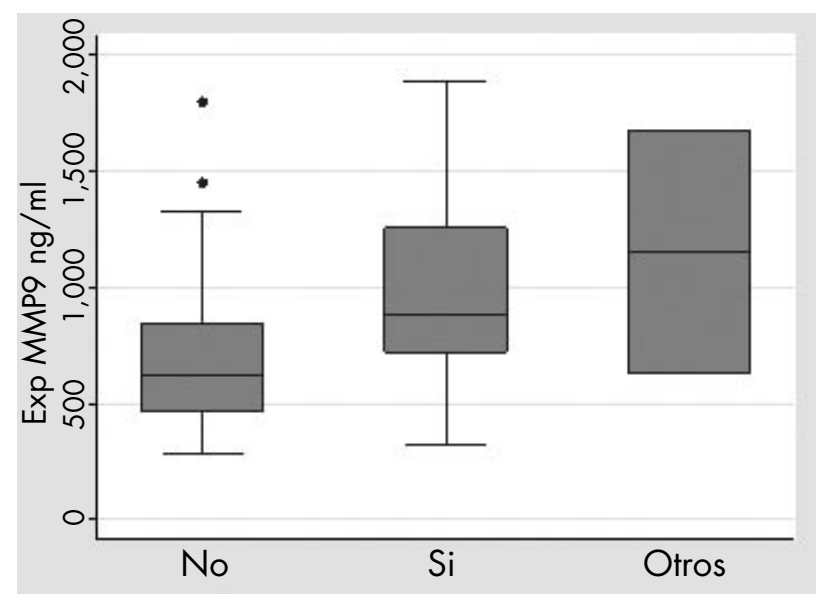

FIGURA 3. Expresión sérica de metaloproteasa 9 en el subgrupo de pacientes con cociente PSA libre entre PSA total menor del 15\%. 
son, no parece existir relación entre los niveles de MMP-9 y el pronóstico, pero esta es una afirmación que debe realizarse con la revisión de los datos a largo plazo.

Finalmente, la relación entre la expresión de MMP-9 y PSA séricos quedó descartada al obtener un coeficiente de correlación de Pearson de $-0,1000$, lo cual aporta un especial interés a este potencial marcador del carcinoma prostático, al ser un marcador cuyos valores son independientes de los niveles PSA.

\section{CONCLUSIONES}

En el presente estudio no ha sido posible demostrar la relación entre la expresión de la metaloproteasa de matriz 9 y el resultado de la biopsia prostática. El análisis por subgrupos muestra una clara tendencia a la significación estadística (sin alcanzarse con este tamaño muestral) en el subgrupo de pacientes con PSA 4-10 ng/ml, y llega a la significación estadística en el subgrupo de PSA libre/total menor del 15\%, aunque este hallazgo tiene una repercusión clínica escasa. Tampoco fue demostrada la asociación entre la expresión de MMP-9 y el grado Gleason. La independencia demostrada entre la expresión de MMP-9 y el PSA podría ser útil en tumores bioquímicamente silentes. Este estudio preliminar presenta una clara limitación por su tamaño muestral, que va a ser ampliado en un futuro inmediato.

\section{BIBLIOGRAFÍA y LECTURAS RECOMENDADAS ( ${ }^{*}$ lectura de interés $y^{* *}$ lectura fundamental)}

**1. Gómez Veiga F, Ponce Reixa J, Barbagelata López A, Fernández Rosado E, González Martín M. Papel actual del Antígeno Prostático Específico y otros marcadores en el Diagnóstico del Cáncer de Próstata. Arch Esp Urol, 2006; 59 (10): 1069-82.

*2. Brawer MK. Prostatic-specific antigen: current status. Cancer J Clin, 1999; 49: 264.

3. Puente X, Sánchez L, Overall C M, and LópezOtín C. Human and mouse proteases: a comparative genomic approach. Nat Rev Genet, 2003; 9: 407-15.

*4. Folgueras AR, Pendás AM, Sánchez LM, LópezOtín C. Matrix metalloproteinases in cancer: From new functions to improved inhibition strategies. Int Dev Biol, 2004; 48: 411-24.

**5. Shafiro SD. Matrix metalloproteinase degradation of extracelular matrix: biological consequences. Curr Opin Cell Biol, 1998; 10: 602-8.
6. Seghal I and Thompson TC. Novel regulation of Type IV Collagenase (Matrix Metalloprotease -9 and -2) activity transforming Growth Factor- $\beta 1$ in Cancer Cll Lines. Mol Biol Cell, 1999; 10(2) 407-16.

7. Grieu F, Qi Li W and Iacopetta B. Genetic polimorfisms in the MMP-2 and the MMP-9 gene and breast cancer phenotype. Breast Cancer res treat 2004; 88 (3): 197-204.

8. Soria de González A, Díaz EI, Guber RS, Arias NN, Ruiz de Martínez N. Niveles séricos de metaloproteasa-9 en mujeres con patologías mamarias. Acta Bioquím. Clín. Latinoam, 2005; 39 (2): 157-61.

9. Hu Z, Huo X, Lu D, Qian J, Zhou J, Chen Y, et al. Functional Polymorphisms of Matrix Metalloproteinase- 9 are associated with Risk of occurrence and metastasis of lung cancer. Clin Cancer Res, 2005; 11 (15): 5433-9.

10. Hueslin MJ, Yna J, Johnson MR, Weiss H, Diasio $\mathrm{RB}$, and Urist MM. Role of Matrix Metalloproteinase in Colorrectal carcinogenesis. Annals of Surg, 2001; 233 (6): 786-92.

11. Kanayama H. Matrix Metalloproteinases and bladder cancer. J Med Invest, 2001; 48: 31-43.

12. Awakura Y, Ito N, Nakamura E, Takahashi T, Kotani H, Mikami Y, et al. Matrix metalloproteinase-9 polymorphisms and renal cell carcinoma in Japanese population. Cancer Letters, 2006; 241: 59-63.

**13. Sauer CG, Kappeler A, Späth M, Kaden JJ, Michel MS, Mayer D, Bleyl, et al. Expresion and activity of matrix metalloproteinases-2 and -9 in serum, core biopsies and tissue specimens of prostate cancer patients. Virchows Arch, 2004; 444 (6): 518-26.

**14. Zhang L, Shi J, Feng J, Klocker H, Lee C, Zhang J. Type IV collagenase (matrix metalloproteinase2 and -9 in prostate cancer. Prostate Cancer Prostatic, Dis 2004; 7 (4): 327-32.

**15. Morgia G, Falsaperla M, Malaponte G, Madonia M, Indelicato M, Travali S, et al. Matrix metalloproteinases as diagnostic (MMP-13) and prognostic (MMP-2, MMP-9) as markers of prostate cancer. Urol Res, 2005; 33 (1): 44-50.

16. Santos Arrontes D, Luján Galán M, Pascual Mateo C, Chiva Robles V, Fernández González I y Berenguer Sánchez A. Análisis descriptivo de los efectos adversos de la biopsia transrectal prostática en 603 procedimientos. Arch Esp Urol, 2004; 57 (6): 601-5.

*17. Naughton CK, Miller DC, Mager DE, Ornstein DK and Catalona WJ. A prospective Randomiced Trial comparing 6 versus 12 prostate biopsy cores: Impact on Cancer Detection. J Urol, 2000; 164: 388-92.

18. Trilla E y. Morote J. Cáncer de Próstata: Nuevas técnicas diagnósticas. Estado actual de la biopsia de próstata. Arch Esp Urol, 2006; 59 (10): 945-52. 